\title{
Morphological and immunohistochemical effect of cerium dioxide nanoparticles on reparative osteogenesis of the jaw bones
}

\author{
Ernest Bazikyan $^{1}$ (D), Andrey Chunikhin ${ }^{*}$ (D), Grigory Volozhin ${ }^{1}$, Knarik Abraamyan ${ }^{1}$, Vladimir Ivanov² (D), Mariya S. Zudina ${ }^{3}$ \\ ${ }^{1}$ Laboratory of Medical Cybernetics and Digital Biomedical Nanotechnology, Moscow State University of Medicine and Dentistry, Moscow, Russian \\ Federation. \\ ${ }^{2}$ Laboratory of Nanobiomaterials and Bioeffectors for Theranostics of Socially Significant Diseases, Kurnakov Institute of General and Inorganic Chemistry \\ Russian Academy of Sciences, Moscow, Russian Federation. \\ ${ }^{3}$ Department of Oral Surgery, Moscow State University of Medicine and Dentistry, Moscow, Russian Federation.
}

\begin{tabular}{l}
\hline ARTICLE INFO \\
\hline Received on: $07 / 08 / 2021$ \\
Accepted on: $25 / 10 / 2021$ \\
Available Online: $05 / 02 / 2022$
\end{tabular}

Key words:

Bone remodeling, cerium oxide nanoparticles, periodontal surgery, platelet-rich plasma.

\begin{abstract}
Several modern scientific studies show that nanodispersed cerium dioxide (nanoCeO ${ }_{2}$ ) provides improved cell proliferation, including osteoblasts, antibacterial, and antiviral effects. In this study, using a comparative morphological, morphometric, and immunohistochemical analysis, we studied the possibilities of the effect of nanoCeO $\mathrm{O}_{2}$ on the processes of bone tissue remodeling after surgical interventions. $\mathrm{NanoCeO}_{2}$ was placed in a new bioconstruction developed by us based on platelet-rich fibrin. Rabbits of the "Soviet Chinchilla" breed were injected with a new biocomposition with nanoCeO $\mathrm{C}_{2}$ on one side of the jaw into a hole formed with a trephine and with granular xenogenic osteoplastic material on the other side. The analysis of the state of the bone tissue was carried out on days $15,25,35,45$, and 55 . The results of the morphological analysis showed that when the defects were filled with a new bioconstruction nanoCeO complete formation of bone tissue was observed, which was almost morphologically identical to the parameters of the native bone tissue. Morphometric analysis showed a high activity of the expression of matrix metalloproteinase- 9 when the defects were filled with a new bioconstruction based on nanoCeO $\mathrm{C}_{2}$ at all stages of observation.
\end{abstract}

\section{INTRODUCTION}

The issues of bone tissue regeneration and remodeling in various pathological conditions or after surgical interventions are a complex problem of dentistry. The process of bone remodeling is mediated by numerous metabolic and immune factors. Undoubtedly, bone cellular elements, such as osteoclasts and osteoblasts, are of great importance in reparative osteogenesis (Marques et al., 2015). The study of the stimulation of reparative osteogenesis using various factors, including the use of the nanostructures of chemical elements and the creation of new materials, does not lose its relevance (Labis et al., 2016). In the early days of dental implantation, various preparations based on

*Corresponding Authotr

Andrey Chunikhin, Laboratory of Medical Cybernetics and Digital Biomedical Nanotechnology, Moscow State University of Medicine and Dentistry, Moscow, Russian Federation.E-mail: docca74@yandex.ru calcium hydroxide were used to stimulate reparative osteogenesis (Goncharov et al., 1996; Khosropanah et al., 2018). In recent years, scientific research has been conducted in the direction of creating tissue-engineered structures using nanodispersed particles of various materials and mesenchymal stem cells and studying their effect on osteoreparative processes in jaw bones during dental implantation (Zhou et al., 2013).

Unique biochemical properties have been found in nanodispersed cerium dioxide (nanoCeO $\mathrm{C}_{2}$ ). Experimental studies in vitro have shown that this material provides an improved proliferation of osteoblasts (Zhou et al., 2013). It has been previously demonstrated that $\mathrm{CeO}_{2}$ nanoparticles have unique antioxidant (Naidi et al., 2021; Shcherbakov et al., 2015), antiinflammatory (Hirst et al., 2009; Li et al., 2020), and radioprotective effects (Popov et al., 2016a, 2016b). They also have the potential to inactivate a wide range of reactive oxygen species (Dowding et al., 2013; Shcherbakov et al., 2015; Shekunova et al., 2019; Xue et al., 2012) due to oxygen nonstoichiometry (Baranchikov et al., 2010; Zholobak et al., 2020). It has also been shown 


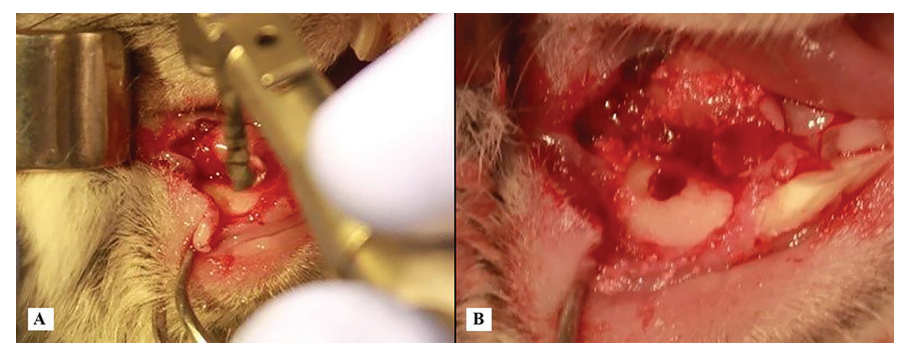

Figure 1. (A) A photograph of the process of preparation of the cavity in the jaw for the experiment. (B) A photograph of a formed bone defect in the jaw.

that $\mathrm{CeO}_{2}$ nanoparticles can perform as the functions of certain oxidoreductases, such as superoxide dismutase and catalase (Heckert et al., 2008; Pirmohamed et al., 2010; Wu et al., 2020).

It seems relevant to develop bioengineering structures based on nanodispersed $\mathrm{CeO}_{2}$ for filling bone defects and stimulating bone tissue regeneration during surgical interventions in the maxillofacial region. It is also important to conduct comparative experimental studies to examine the possible effects of nanodispersed $\mathrm{CeO}_{2}$ as part of new biocomplexes on the processes of bone tissue remodeling. Morphometric and immunohistochemical analysis in vivo should be used.

\section{MATERIALS AND METHODS}

\section{Animals}

The study was conducted in accordance with the Animal Research: Reporting of In Vivo Experiments guidelines, the rules for working with animals (based on the provisions of the Declaration of Helsinki and the recommendations contained in the EU Directive 86/609/ECC and the European Convention for the Protection of Vertebrate Animals Used for Experimental and Other Scientific Purposes). Also, we obtained the permission of the Interuniversity Ethics Committee of the Moscow State University of Medicine and Dentistry (protocol N 07-19 dated 07/18/2019). Twenty sexually mature male rabbits of the "Soviet Chinchilla" breed weighing 2.5-3.0 $\mathrm{kg}$ were used. A soft tissue incision up to $2 \mathrm{~cm}$ in length was made along the lower edge of the mandible. This was carried out under general anesthesia by intramuscular injection of Xylazine $(4-6 \mathrm{mg} / \mathrm{kg})$ and Zoletil $100(5-10 \mathrm{mg} / \mathrm{kg})$ to provide analgesia and relaxation for $30-40$ minutes. Then, a hole with a diameter of $4-5 \mathrm{~mm}$ and a depth of about $4 \mathrm{~mm}$ was dissected in the jawbone using a bur of the appropriate diameter and cooling with saline (Fig. 1A and B).

In accordance with the study, the jawbones of the animals were dissected on both sides of the jaw. On the left

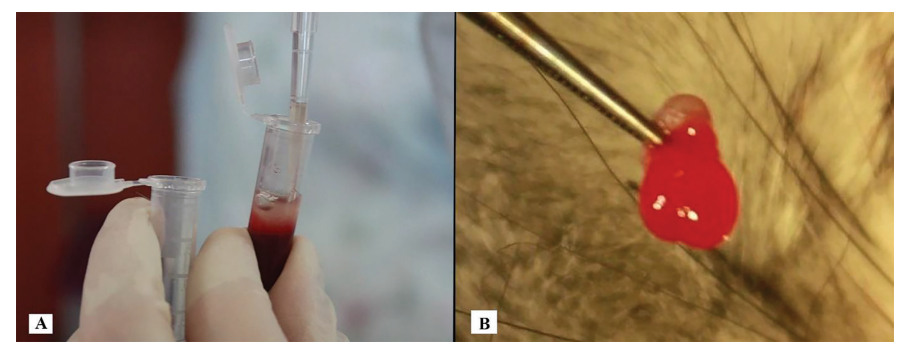

Figure 3. (A) A photograph of PRF supernatant selection for the addition of nanoCeO ${ }_{2}$. (B) A photograph of the formed $\mathrm{PRF}$ nanoCeO $\mathrm{C}_{2}$ clot after the addition of Thrombin and Renamplatin.

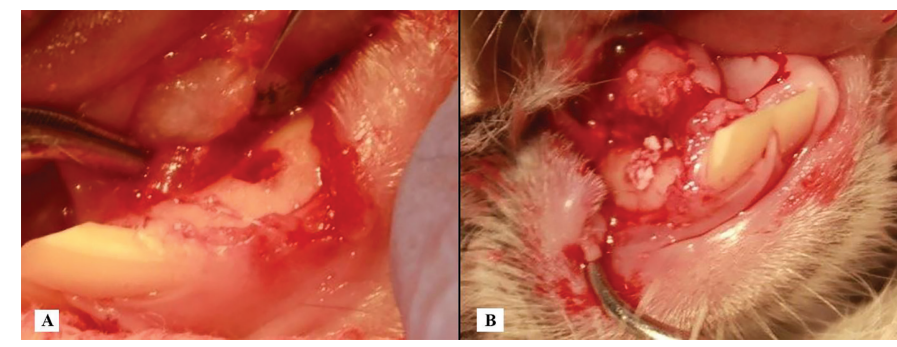

Figure 2. (A) A photograph of the introduction of a new PRF biocomposition into the formed bone defect based on nanoCeO $\mathrm{C}_{2}$. (B) A photograph of the introduction of xenogeneic granules into the defect.

(the experiment side), a new biocomposition based on plateletrich fibrin (PRF) saturated with cerium dioxide nanoparticles was placed in the formed bone defect. On the right (the comparison side), the defect was filled with granules of xenogenic osteoplastic material (XOM). The wounds were sutured on both sides using a bioresorbable suture (Fig. 2A and B).

\section{Obtaining PRF with nano-CEO}

Blood was collected from the lateral vein of the rabbit's ear. For this purpose, a $5 \mathrm{ml}$ syringe and a $23 \mathrm{G}$ needle, previously soaked in sodium citrate solution, were used. Then, tubes containing 3.8\% sodium citrate were filled with $4 \mathrm{ml}$ of blood each. Citrate-stabilized blood was transferred to $1.5 \mathrm{ml}$ Eppendorf tubes and centrifuged at 1,000 rpm for 10 minutes. After centrifugation, the supernatant was taken into a new tube and recentrifuged at 1,500 rpm for 10 minutes (Fig. 3A).

After repeated centrifugation, the platelet-free plasma was carefully removed to obtain $0.5 \mathrm{ml}(500 \mu \mathrm{l})$ of platelet-rich plasma (PRP). $50 \mu \mathrm{l}$ of cerium suspension was added to $500 \mu \mathrm{l}$ of PRP and gently mixed. Plasma with cerium was supplemented with $100 \mu \mathrm{l}$ of Thrombin (in the form of a suspension with a concentration of $2 \mathrm{mg} / \mathrm{ml}$ ) and with $100 \mu \mathrm{l}$ of Renamplastin (which is a thromboplastin with factor III and calcium ions). The formation of a clot took place within 1 minute and its contraction within another 1 minute. The finished clot, represented by PRF saturated with nanoCeO, was carefully squeezed out in a sterile Petri culture plate. It was then introduced into the bone hole (Fig. 3B).

We used a sol of polymer microcapsules of citrate nanoCeO2 in saline Parg/DS/Parg/DS/Parg/citrate nanoCeO $/ \mathrm{Parg} /$ DS, where Parg is poly-L-arginine hydrochloride mol wt 15,000 70,000 ; DS is dextran sulfate sodium salt mol wt 40,000 , obtained according to the method described by Ivanov et al. (2010) and

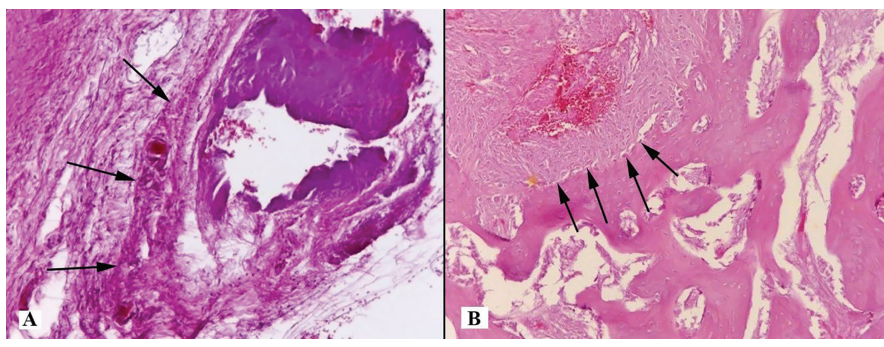

Figure 4. Histotopogram of the cross section of the rabbit jaw on the 15th day of the experiment: (A) proliferation of full-blooded connective tissue (arrows); (B) a large number of osteoblasts (arrows) (hematoxylin-eosin, Zeiss, $\times 120$ ). 
Nikolaeva et al. (2020). The study was conducted in accordance with the protocol recommended by ISO (ISO 7405: 1997).

\section{Histological examination}

All the animals were euthanized with a lethal intravenous dose of Zoletil $100(50 \mathrm{mg} / \mathrm{kg})$. Four animals were taken out of the experiment on each of the days $15,25,35,45$, and 55 . Decapitation was carried out. The specimens were then fixed in a $10 \%$ buffered formalin solution, decalcified according to the standard method with reagent Biodek R (Biodek, Aprilia, Lazio, Italy), and then embedded in paraffin blocks. Histological sections, $4 \mu \mathrm{m}$ thick, obtained on an HM355S microtome (Thermo Fisher Scientific, Hillsboro, OR), were stained with hematoxylin and eosin. Descriptive microscopic analysis was carried out on an AxioLab A1 microscope (Carl Zeiss Microscopy, Oberkochen, Germany) by three laboratory technicians on samples distributed between them in a blinded manner. The description of the nature of tissues in the groups was made according to the following parameters: the presence or absence of newly formed bone trabeculae, plethora of blood vessels, osteoblasts, areas of bone tissue necrosis, and the thickness and the restorative nature of changes in the bone tissue.

\section{Immunohistochemical Research}

After high-temperature recovery of antigenic determinants in the PT-module for thermal treatment of paraffin sections (Thermo Fisher Scientific, Hillsboro, OR), immunohistochemical reactions were carried out. This was carried out in an autostainer (Thermo Fisher Scientific, Hillsboro, OR) according to the protocols supplied by the manufacturers to monoand polyclonal antibodies. To visualize the reactions, we used a ready-made test system with universal secondary antibodies labeled with a chromogen (3,3'-diaminobenzidine), Histophine (Nichirei Corp., Chūō, Tokyo, Japan). Two negative controls (for the specificity of the reaction and the absence of active endogenous peroxidase) and one internal positive control (for the specificity of the reaction) were taken into account. Antibodies to matrix metalloproteinase (MMP)-9 and tissue inhibitors of metalloproteinase (TIMP)-1 (Spring Bioscience Corporation, Pleasanton, CA) were used as primary antibodies.

\section{Method of statistical data processing}

To evaluate the results of the reaction, a routine semiquantitative method was used (according to the degree of expression from 0 to 3 points) (Cregger et al., 2006). The calculation was carried out in 10 randomly selected fields of view

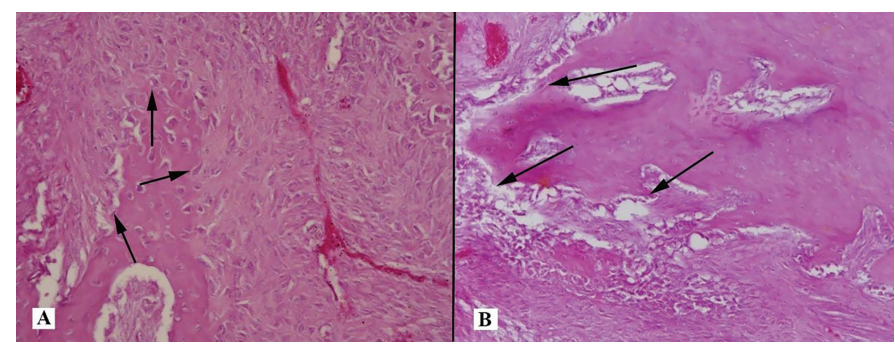

Figure 5. Histotopogram of the cross section of the rabbit jaw on the 25th day of the experiment: (A) a large number of osteoblasts (arrows); (B) proliferation of bone trabeculae surrounded by a large number of osteoblasts (arrows) (hematoxylin-eosin, Zeiss, $\times 120$ ). at an $120 \times$ magnification of the microscope. The results were statistically processed using MS Excel and Statistica for Windows 10.0 software. They were then presented as a mean value \pm standard deviation $(M \pm \sigma)$. Also, the method of nonparametric analysis according to Mann and Whitney (1947) was used (Mann and Whitney, 1947). The value of the critical significance level was assumed to be 0.05 ; that is, differences were considered as statistically significant at $p<0.05$.

\section{RESULTS}

\section{Histological analysis}

Fifteen days later, a histological assessment of the bone tissue of the right jaw (the comparison side) was carried out. It showed the presence of encapsulated basophilic structureless masses surrounded by overgrowths of full-blooded connective tissue among partially destroyed and necrotic trabeculae of cancellous bone (Fig. 4A). In the left jaw (the experiment side), there was a focal proliferation of connective tissue with a pronounced plethora of blood vessels in the thickness of the cancellous bone. At the border with the bone trabeculae, there were accumulations of osteoblasts with an admixture of osteoclasts (Fig. 4B).

On the 25th day of the experiment, in the defect filled with osteoplastic material, there was a focal proliferation of connective tissue in the thickness of the cancellous bone. We also observed a pronounced plethora of blood vessels, small basophilic and partially lysed acellular fragments, with a growth of new bone trabeculae surrounded by a large number of osteoblasts (Fig. 5A). On the side of the experiment, where the defect was filled with PRF saturated with nanoCeO ${ }_{2}$, there was a focal proliferation of bone trabeculae in the thickness of the cancellous bone in the connective tissue. The bone trabeculae were surrounded by a large number of osteoblasts with single osteoclasts (Fig. 5B).

On the 35th day of the experiment, focal formation of bone trabeculae was observed in the thickness of the cancellous bone in the full-blooded connective tissue on the comparison side. These bone trabeculae formed an area of compact bone tissue with single small sequesters and pronounced lines of osteon boundaries surrounded by a large number of osteoblasts with an admixture of osteoclasts (Fig. 6A). On the side of the experiment, the main difference was the absence of sequestration and the formation of bone trabeculae surrounded by a large number of osteoblasts with single osteoclasts (Fig. 6B).

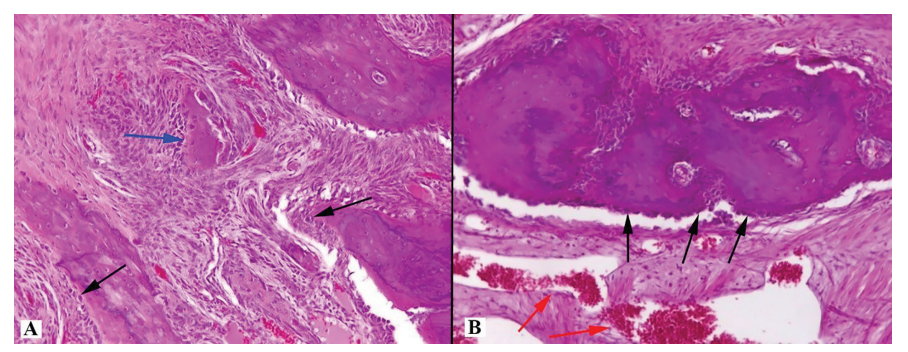

Figure 6. Histotopogram of the cross section of the rabbit jaw on the 35th day of the experiment: (A) single small sequestration (blue arrow) and pronounced lines of the boundaries of osteons surrounded by a large number of osteoblasts (black arrows); (B) a large number of osteoblasts (black arrows) and vascular congestion (red arrows) (hematoxylin-eosin, Zeiss, $\times 120$ ). 


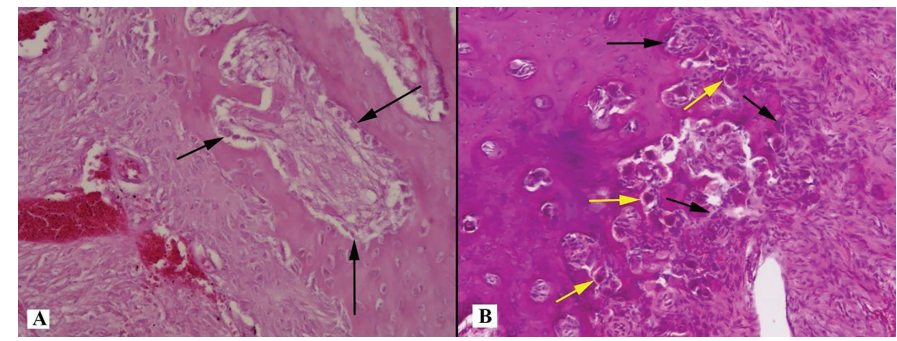

Figure 7. Histotopogram of the cross section of the rabbit jaw on the 45th day of the experiment: (A) pronounced lines of the boundaries of osteons surrounded by a large number of osteoblasts (arrows); (B) formation of bone trabeculae surrounded by a large number of osteoblasts (black arrows) and osteoclasts (yellow arrows) (hematoxylin-eosin, Zeiss, $\times 120$ ).

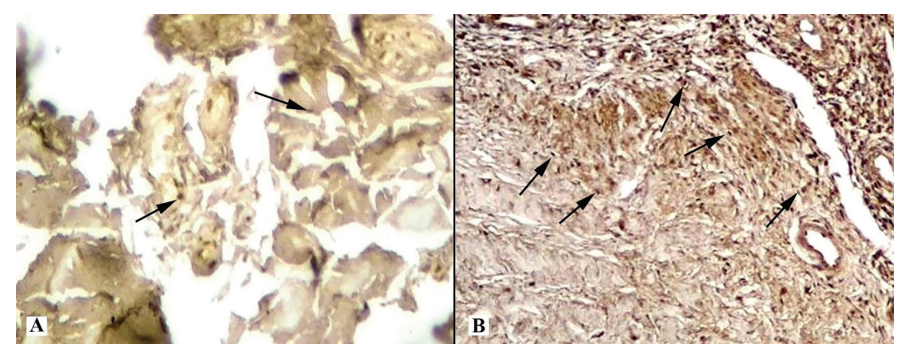

Figure 9. Histotopogram of the cross section of the rabbit jaw on the 15th day of the experiment: (A) introduction of xenogeneic granules into the formed bone defect, weak expression of MMP-9 (arrows); (B) introduction of a new PRF biocomposition into the defect based on nanoCeO $\mathrm{C}_{2}$, pronounced expression of MMP-9 (arrows) (Mayer's hematoxylin, Zeiss, $\times 120$ ).

On the 45th day of the experiment, histologically, there was the focal formation of bone trabeculae in the thickness of the cancellous bone, the full-blooded connective tissue on the comparison side. These bone trabeculae formed an area of compact bone tissue, with pronounced lines of osteon boundaries surrounded by a large number of osteoblasts with single osteoclasts. Granuloma-like structures were also found (Fig. 7A). On the side of the experiment, the main histological difference was the focal formation of bone trabeculae surrounded by a large number of osteoblasts and osteoclasts and the absence of granuloma-like structures (Fig. 7B).

On the 55th day, formed compact bone tissue was observed in the area of surgery with the introduction of granular osteoplastic xenogenic material into the cavity of the defect. This bone tissue corresponded in structure with the cortical plate,

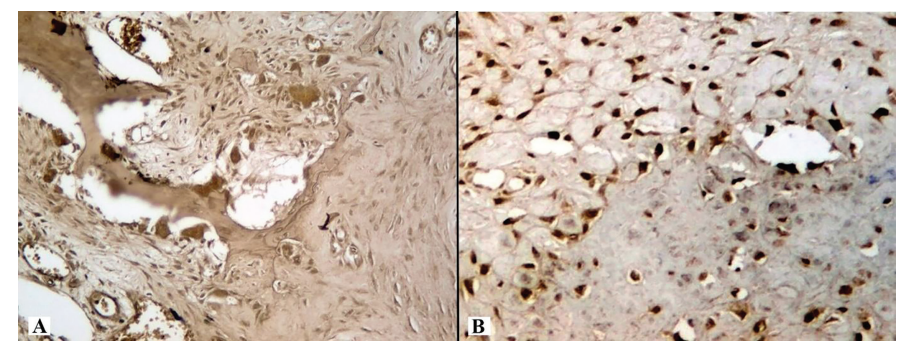

Figure 11. Histotopogram of the cross section of the rabbit jaw on the 35th day of the experiment: (A) introduction of xenogeneic granules into the formed bone defect, pronounced expression of MMP-9, especially in osteoclasts; (B) introduction of a new PRF biocomposition into the defect based on nanoCeO, pronounced expression of MMP-9 (Mayer's hematoxylin, Zeiss, $\times 120$ ).

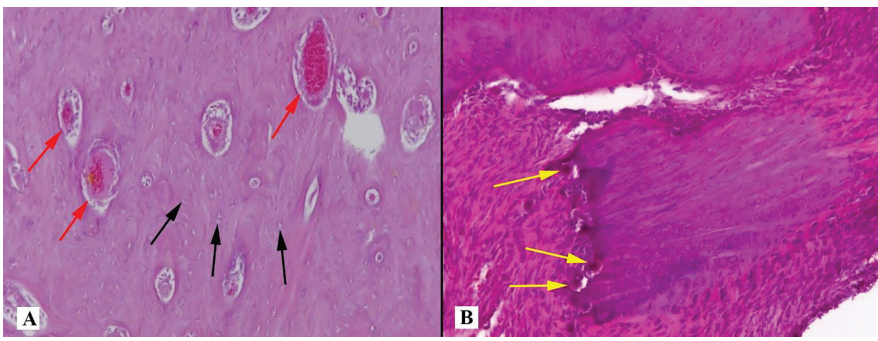

Figure 8. Histotopogram of the cross section of the rabbit jaw on day 55 of the experiment: (A) compact bone tissue with moderately dilated and plentiful lacunae (red arrows), in which there are many osteoblasts (black arrows); (B) areas of the spongy structure that are locally surrounded by osteoclasts (yellow arrows) (hematoxylin-eosin, Zeiss, $\times 120$ ).

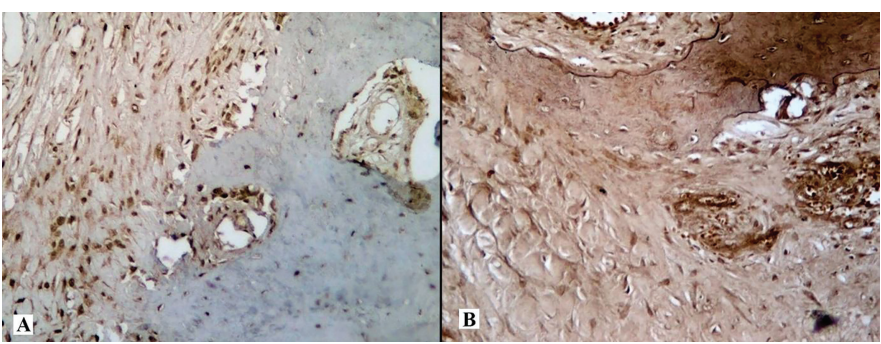

Figure 10. Histotopogram of the cross section of the rabbit jaw on the 25th day of the experiment: (A) introduction of xenogeneic granules into the formed bone defect, pronounced expression of MMP-9; (B) introduction of a new PRF biocomposition into the defect based on nanoCeO $\mathrm{C}_{2}$, pronounced expression of MMP-9 (Mayer's hematoxylin, Zeiss, $\times 120$ ).

with moderately dilated and full-blooded lacunae, in which there were many osteoblasts (Fig. 8A). At the same time, there were areas of a cancellous structure on the side of the defect filled with PRF saturated with nanoCeO $\mathrm{O}_{2}$. These areas showed proliferation of connective tissue between the bone trabeculae, which were sometimes surrounded by osteoclasts (Fig. 8B).

\section{Immunohistochemical analysis}

MMPs belong to the family of zinc metalloproteinases. Their function is associated with the metabolism of the connective tissue matrix of the bone in normal and pathological. MMP activity is regulated by both nonspecific inhibitors, $\alpha 2-$ macroglobulins, and specific inhibitors, TIMP. The positive results of the immunohistochemical reaction were taken into account with two negative controls and one internal positive

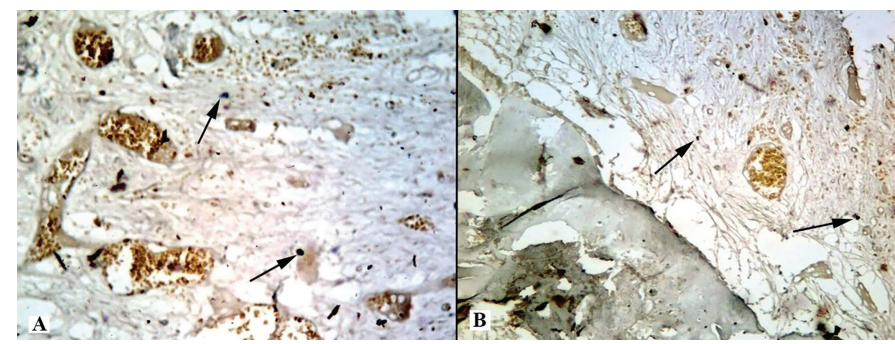

Figure 12. Histotopogram of the cross section of the rabbit jaw on the 45th day of the experiment: (A) introduction of xenogeneic granules into the formed bone defect, weak expression of TIMP-1 (arrows); (B) introduction of a new PRF biocomposition into the defect based on nanoCeO ${ }_{2}$, weak expression of TIMP-1 (arrows) (Mayer's hematoxylin, Zeiss, $\times 120$ ). 


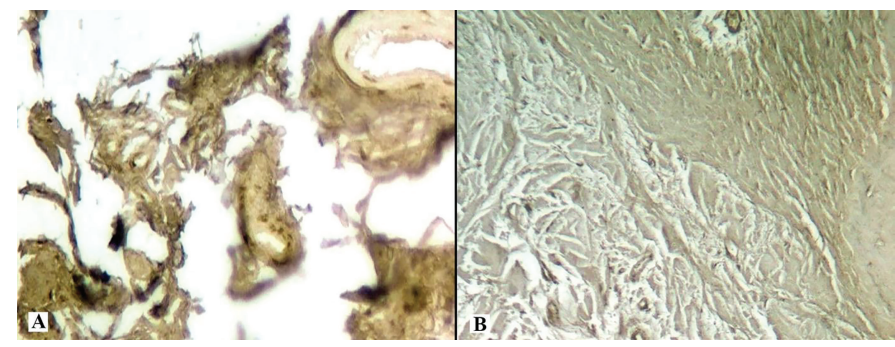

Figure 13. Histotopogram of the cross section of the rabbit jaw on the 45th day of the experiment: (A) introduction of xenogeneic granules into the formed bone defect, weak expression of MMP-9; (B) introduction of a new PRF biocomposition into the defect based on nanoCeO ${ }_{2}$, weak expression of TIMP-1 (Mayer's hematoxylin, Zeiss, $\times 120$ ).

control present. The negative controls were on the specificity of the reaction and the absence of active endogenous peroxidase. The internal positive control was on the specificity of the reaction. Immunograms show the expression of MMP-9 and TIMP-1 (Figs. 9-13).

On the comparison side, the maximum expression of MMP-9 and the minimum expression of TIMP-1 were detected on days 25-35. The expression of TIMP-1 increased on the 45 th day, and the ratio changed by the 55th day: the expression of TIMP-1 significantly increased, whereas the expression of MMP-9 decreased. On the side with PRF implantation into the bone defect with nanoCeO ${ }_{2}$, the highest expression of MMP-9 and the lowest expression of TIMP-1 were noted already from the 15 th day. This ratio remained unchanged on the 25 th -35 th days and changed on the 45 th-55th days. It remained higher compared to the other side with the introduction of granular osteoplastic material.

\section{Morphometric analysis}

The results of a morphometric study of the expression of MMP-9 and TIMP-1 showed the following. Upon implantation of a granular osteoplastic xenogenic material into the defect area, production in the MMP-9 tissue is maximal on the 25th-35th days. Upon the introduction of PRF saturated with nanoCeO, production in the MMP-9 tissue is maximal on the 15th-35th days. It becomes elevated already on the 15 th day and remains high after 45 days (Table 1).

The expression of MMP-9 and TIMP-1 in the foci of implantation of granular osteoplastic material and PRF with nanoCeO $\mathrm{C}_{2}$ into the bone defect on different days (15-55) of the experiment is shown in Figures 14 and 15.

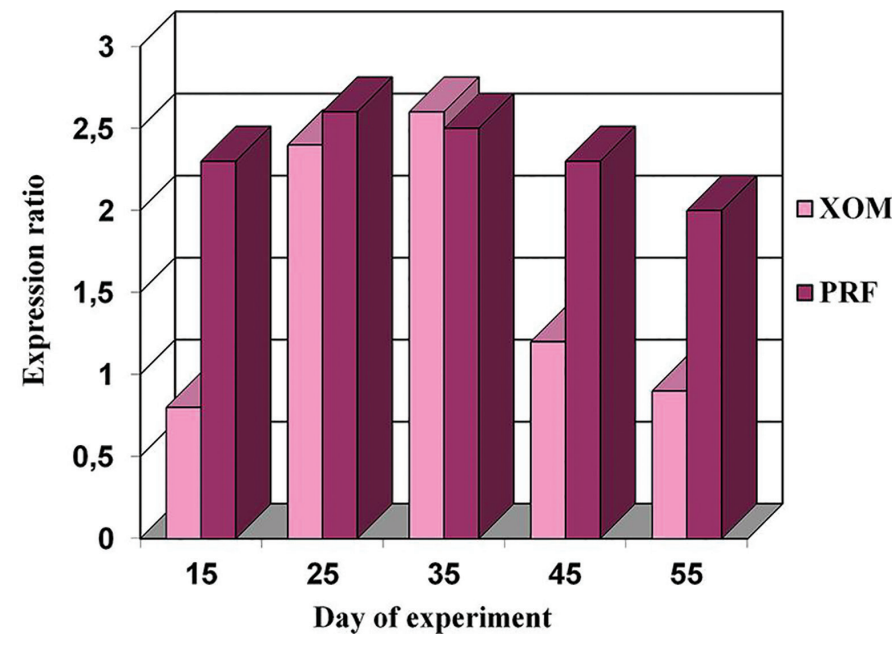

Figure 14. Expression of MMP-9 in the foci of implantation of XOM and PRF with nanoCeO ${ }_{2}$ on different days $(15-55)$ of the experiment.

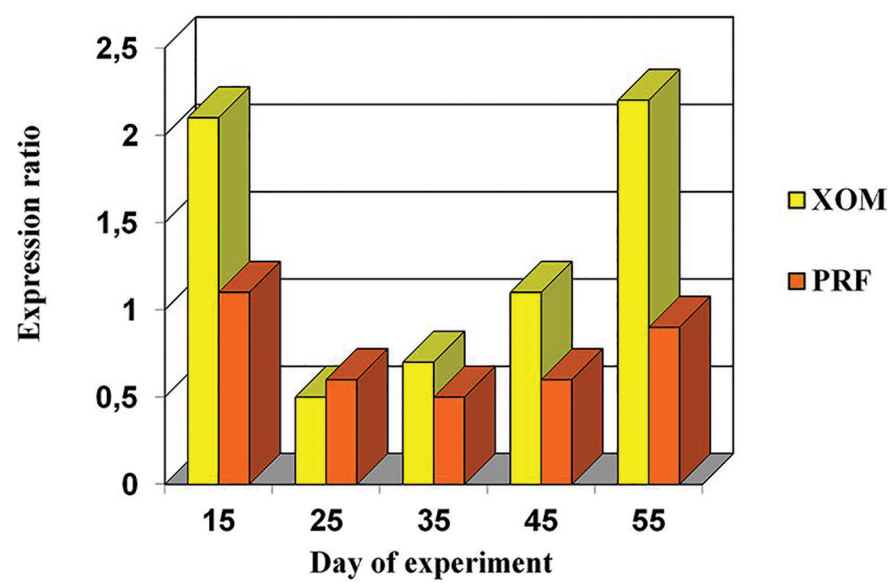

Figure 15. Expression of TIMP-1 in the foci of implantation of XOM and PRF with nanoCeO $\mathrm{O}_{2}$ on different days (15-55) of the experiment.

\section{DISCUSSION}

Theobtainedresults of themorphological,morphometric, and immunohistochemical analysis demonstrated significant differences in the process and result of bone tissue formation on different sides of the jaw. The presence of nanoCeO in the new bioconstruction based on PRF proved that there is a significant effect on the rate and quality of jaw bone tissue regeneration. Our results are consistent with the results of modern research. This research has established in vitro in laboratory conditions

Table 1. Expression of MMP-9 and TIMP-1 $(M \pm \sigma)$ in the foci of implantation of XOM (1) and PRF with nanoCeO $\mathrm{O}_{2}(2)$ at different times of the experiment.

\begin{tabular}{cccccccccccc}
\hline $\begin{array}{c}\text { Day of observation } \\
\text { Indicator/material }\end{array}$ & $\mathbf{1}$ & $\mathbf{2}$ & $\mathbf{1}$ & $\mathbf{2}$ & $\mathbf{1}$ & $\mathbf{2}$ & $\mathbf{4}$ & $\mathbf{4 5}$ & $\mathbf{2}$ & $\mathbf{1}$ & $\mathbf{2}$ \\
\hline MMP-9 & $0.8 \pm 0.73$ & $2.3 \pm 0.48^{*}$ & $2.4 \pm 0.57$ & $2.6 \pm 0.50$ & $2.6 \pm 0.42$ & $2.5 \pm 0.31$ & $1.2 \pm 0.11$ & $2.3 \pm 0.27^{*}$ & $0.9 \pm 0.11$ & $2.0 \pm 0.29^{*}$ \\
TIMP-1 & $2.1 \pm 0.33$ & $1.1 \pm 0.51^{*}$ & $0.5 \pm 0.12$ & $0.6 \pm 0.16$ & $0.7 \pm 0.09$ & $0.5 \pm 0.08$ & $1.1 \pm 0.10$ & $0.6 \pm 0.07^{*}$ & $2.2 \pm 0.20$ & $0.9 \pm 0.06^{*}$ \\
\hline
\end{tabular}

The difference is statistically significant $(p=0.00342)$ between the indicators in the groups.

* Indicates $\mathrm{ng} / \mathrm{ml}$. 
that $\mathrm{CeO}_{2}$ nanoparticles promote proliferation and differentiation of osteoblasts without cytotoxic effects on tissues (Popov et al., 2016a, 2016b; Purohit et al., 2020; Shcherbakov et al., 2011). In our study, we used a standard granular osteoplastic material and a new bioengineering design based on nanoCeO $\mathrm{C}_{2}$. As a result of our study, significant morphological, morphometric, and immunohistochemical differences in the formation of bone tissue on different sides of the jaw were revealed at different stages of observation. On the 35th day of observation, in defects filled with a new construction based on nanoCeO ${ }_{2}$, the formation of cancellous bone trabeculae was observed, surrounded by a large number of osteoblasts. In contrast, in defects filled with XOM, focal formation of bone trabeculae of compact bone was noted at this stage of observation. By the 55th day of observation, complete formation of full-fledged bone tissue was noted in the thickness of the defect filled with a new bioconstruction based on nanoCeO ${ }_{2}$. This bone tissue was almost identical in its morphological parameters to native bone tissue. In defects filled with granular osteoplastic material, the formation of compact bone tissue was noted, similar in its structure to the cortical bone. Morphometric analysis showed the activity of MMP-9 expression on the side of defects filled with a new bioconstruction based on nanoCeO $\mathrm{O}_{2}$ at all stages of observation. Starting from the 15th day of observation, the activity of MMP9 increased significantly. It remained at a sufficiently high level up to the 55th day $(2.3 \pm 0.48$ and $2.0 \pm 0.29$, resp. $)$. In defects filled with granules of XOM, the expression of MMP-9 was significantly lower $(0.8 \pm 0.73)$ by the 15 th day, which is by 2.88 times compared with the main group. It increased only on the 25 th day of the experiment $(2.4 \pm 0,57)$. By the 45 th -55 th day of observation, it significantly decreased $(0.9 \pm 0.11)$, almost by 2.2 times compared to the main group. At the same time, TIMP-1 expression was determined at a low level at all stages of observation in the main group, from the 15th $(1.1 \pm 0.51)$ to the 55th day of observation $(0.9 \pm 0.06)$. In the control group, these indicators showed significant differences. On the 15th day, TIMP-1 expression was 1.9 times higher $(2.1 \pm 0.33)$. On the 55th day, it was 2.4 times higher $(2.2 \pm 0.20)$ compared to the main observation group. The data obtained indicate a prolonged effect of nanoCeO $\mathrm{C}_{2}$ as part of a PRF-based bioconstruction on reparative osteogenesis at all stages of osteoregeneration. In defects filled with a new bioconstruction based on nanoCeO ${ }_{2}$, the processes of bone tissue regeneration are intensified. A larger volume of bone tissue is formed, morphologically identical to the native bone. The amount of granulation tissue decreases. All this indicates a stimulating effect of nanoCeO $\mathrm{O}_{2}$ on the processes of bone tissue regeneration. The stimulating effect of nanoCeO on the stimulation of differentiation and proliferation of osteoblasts is confirmed by modern in vitro studies (Zhou et al., 2013). In addition, other studies have shown that nanoCeO $\mathrm{C}_{2}$ helps to reduce inflammatory responses by inhibiting interleukin 1-alpha (Ponnunrangam et al., 2014).

The authors plan to continue research in vivo to study the effect of nanoCeO $\mathrm{O}_{2}$ on bone tissue regeneration by regulating redox processes in tissues. Also, ways of affecting inflammatory interleukins, which have a significant effect on bone tissue regeneration, will be studied. The authors plan to continue the development of new biostructures based on nanoCeO $\mathrm{Ce}_{2}$.

\section{CONCLUSION}

The morphological and immunohistochemical studies carried out by the authors have shown that, after the implantation of granular osteoplastic xenogenic material, a connective tissue focus of a larger size is formed. It contains basophilic masses and small fragments of necrotic bone trabeculae (sequesters). The latter persist up to the 35 th-45th days. On the 45th day, granuloma-like structures remain in their place. The peak of osteoblast proliferation occurs on the 25th-35th day. By the 45 th day, the processes of osteosynthesis and bone remodeling predominate. On the 55th day, a newly formed bone tissue is formed. This bone tissue is close in structure to the compact bone of the cortical plate.

After the implantation of PRF with nanoCeO $\mathrm{C}_{2}$ into a bone defect, the volume of newly formed connective tissue is much smaller. Bone sequesters and basophilic material are not found. Earlier, already from the 15th day, there is a pronounced proliferation of osteoblasts. Their peak falls on the 15th-25th day, but it stretches up to the 45th day. The processes of osteosynthesis and bone remodeling do not stop by the 55th day. As a result, bone areas are formed, close in structure to both compact and cancellous bone tissue.

Thus, according to the results of the studies conducted, it has been proved that the introduction of biostructures based on nanodispersed $\mathrm{CeO}_{2}$ as part of PRF into the bone defect promotes acceleration of regeneration due to the stimulation of osteoblastogenesis and neovascularization. All this indicates the stimulation of full-fledged processes of bone tissue regeneration of bone tissue, morphologically identical to the native bone of the jaw.

\section{ACKNOWLEDGMENTS}

This article does not include research involving humans or animals by anyone other than the authors.

\section{CONFLICTS OF INTERESTS}

The authors declare that there is no conflict of interest.

\section{FUNDING}

This research is subsidized by the Ministry of Health of the Russian Federation No. 056-00035-21-00 dated 12/17/2020.

\section{ETHICAL APPROVAL}

This research had ethical approval by the Research Ethics Commission of the Ethics Committee Moscow State University of Medicine and Dentistry, Moscow, Russian Federation (protocol N 07-19 dated 07/18/2019).

\section{AUTHORS' CONTRIBUTIONS}

All authors made substantial contributions to conception and design, acquisition of data, or analysis and interpretation of data; took part in drafting the article or revising it critically for important intellectual content; agreed to submit to the current journal; gave final approval of the version to be published; and agreed to be accountable for all aspects of the work. All the authors are eligible to be an author as per the international committee of medical journal editors (ICMJE) requirements/guidelines. 


\section{PUBLISHER'S NOTE}

This journal remains neutral with regard to jurisdictional claims in published institutional affiliation.

\section{REFERENCES}

Baranchikov AE, Polezhaeva OS, Ivanov VK, Tretyakov YD. Lattice expansion and oxygen non-stoichiometry of nanocrystalline ceria. Cryst Eng Comm, 2010; 12:3531-3.

Cregger M, Berger AJ, Rimm DL. Immunohistochemistry and quantitative analysis of protein expression. Arch Pathol Lab Med, 2006; 130:1026-30.

Dowding JM, Seal S, Self WT. Cerium oxide nanoparticles accelerate the decay of peroxynitrite (ONOO-). Drug Deliv Transl Res, 2013; 3:375-9.

Goncharov, Bazikian EA, Bychkov AI. The use of hydroxyapol in filling in bone defects of the jaws and stimulating osteogenesis. Stomatologiia, 1996; 75:54-6.

Heckert EG, Karakoti AS, Seal S, Self WT. The role of cerium redox state in the SOD mimetic activity of nanoceria. Biomaterials, 2008; 29:2705-9.

Hirst SM, Karakoti AS, Tyler RD, Sriranganathan N, Seal S, Reilly CM. Anti-inflammatory properties of cerium oxide nanoparticles. Nanotherapeutics, 2009; 5:2848-56.

Ivanov VK, Polezhaeva OS, Shaporev AS, Baranchikov AE, Shcherbakov AB, Usatenko AV. Synthesis and thermal stability of nanocrystalline ceria sols stabilized by citric and polyacrylic acid. Russ J Inorg Chem, 2010; 55:328-32.

Khosropanah H, Lashkarizadeh N, Ayatollahi M, Kaviani M, Mostafavipour Z. The impact of calcium hydroxide on the osteoinductive capacity of demineralized freeze-dried bone allograft: an in-vitro study. J Dent (Shiraz), 2018; 1:19-27.

Labis VV, Bazikyan EA, Kozlov IG, Sizova SV, Khaidukov SV. Nanosized particles are participants of osseointegration. Bull Orenburg Sci Center UrO RAN, 2016; 1:5.

Li H, Xia P, Pan S, Qi Z, Fu C, Yu Z, Kong W, Chang Y, Wang $\mathrm{K}$, Wu D, Yang $\mathrm{X}$. The advances of ceria nanoparticles for biomedical applications in orthopaedics. Int J Nanomed, 2020; 15:7199-214.

Mann HB, Whitney DR. On a test of whether one of two random variables is stochastically larger than the other. Ann Math Stat, 1947; 18:50-60.

Marques LF, Stessuk T, Camargo IC, Sabeh Junior N, dos Santos L, Ribeiro-Paes JT. Platelet-rich plasma (PRP): methodological aspects and clinical applications. Platelets, 2015; 26:101-13.

Naidi SN, Harunsani MH, Tan AL, Khan MM. Green-synthesized $\mathrm{CeO}(2)$ nanoparticles for photocatalytic, antimicrobial, antioxidant and cytotoxicity activities. J Mater Chem B, 2021; 9:5599-620.

Nikolaeva AL, Gofman IV, Yakimansky AV, Ivankova EM, Gulii NS, Teplonogova MA, Ivanova OS, Baranchikov AE, Ivanov VK. Interplay of polymer matrix and nanosized redox dopant with regard to thermooxidative and pyrolytic stability: $\mathrm{CeO} 2$ nanoparticles in a milieu of aromatic polyimides. Mater Today Commun, 2020; 22:100803.
Pirmohamed T, Dowding JM, Singh S, Wasserman B, Heckert E, Karakoti AS, King JE, Seal S, Self WT. Nanoceria exhibit redox statedependent catalase mimetic activity. Chem Commun, 2010; 46:2736-8.

Ponnunrangam S, O'Connel GD, Chernyshova IV, Wood K, Hung CT, Ponisseril S. Beneficial effects of cerium oxide nanoparticles in development of chondrocyte-seeded hydrogel constructs and cellular response to interleukin insults. Tissue Eng Part A, 2014; 20:2908-19.

Popov AL, Popova NR, Ivanov VK. Cerium dioxide nanoparticles are not toxic to human MSCs and are capable of stimulating their proliferation in vitro. Topical Issues Biol Phys Chem, 2016a; 2:47-51.

Popov AL, Zaichkina SI, Popova NR, Rozanova OM, Romanchenko SP, Smirnov AA, Mironova EA, Selezneva II, Ivanova OS, Ivanov VK. Radioprotective effect of ultra-small citrate-stabilized cerium oxide nanoparticles in vitro and in vivo. RSC Adv, 2016b; 6:106141-9.

Purohit SD, Singh H, Bhaskar R, Yadav I, Chou CF, Gupta MK, Mishra NC. Gelatin-alginate-cerium oxide nanocomposite scaffold for bone regeneration. Mater Sci Eng C Mater Biol Appl, 2020; 116:111111.

Shcherbakov AB, Zholobak NM, Ivanov VK, Tretyakov YD, Spivak NY. Cerium dioxide nanomaterials: properties and prospects for use in biology and medicine. Biotechnology, 2011; 4:9-28.

Shcherbakov AB, Zholobak NM, Spivak NY, Ivanov VK Advances and prospects of using nanocrystalline ceria in prolongation of lifespan and healthy aging. Russ J Inorg Chem, 2015; 60:1595-625.

Shekunova TO, Lapkina LA, Shcherbakov AB, Meshkov IN, Ivanov VK, Tsivadze AY, Gorbunova Y. Deactivation of singlet oxygen by cerium oxide nanoparticles. J Photochem Photobiol A Chem, 2019; $382: 111925$

Wu L, Liu G, Wang W, Liu R, Liao L, Cheng N, Li W, Zhang W, Ding D. Cyclodextrin-modified $\mathrm{CeO}(2)$ nanoparticles as a multifunctional nanozyme for combinational therapy of psoriasis. Int J Nanomed, 2020; $15: 2515-27$.

Xue Y, Zhai Y, Zhou K, Wang L, Tan H, Luan Q, Yao X. The vital role of buffer anions in the antioxidant activity of $\mathrm{CeO} 2$ nanoparticles. Chemistry, 2012; 18:11115-22.

Zholobak NM, Shcherbakov AB, Ivanova OS, Reukov V, Baranchikov AE, Ivanov VK. Nanoceria-curcumin conjugate: synthesis and selective cytotoxicity against cancer cells under oxidative stress conditions. J Photochem Photobiol B, 2020; 209:111921.

Zhou G, Gu G, Li Y, Zhang Q, Wang W, Wang S, Zhang J. Effects of cerium oxide nanoparticles on the proliferation, differentiation, and mineralization function of primary osteoblasts in vitro. Biol Trace Elem Res, 2013; 153:411-8.

How to cite this article:

Bazikyan E, Chunikhin A, Volozhin G, Abraamyan K, Ivanov $\mathrm{V}$, Zudina MS. Morphological and immunohistochemical effect of cerium dioxide nanoparticles on reparative osteogenesis of the jaw bones. J Appl Pharm Sci, 2022; 12(02):165-171. 